\title{
Development and application of dot-enzyme-linked immunosorbent (dot-ELISA) assay for detection of Brucella melitensis and evaluation of the shedding pattern in infected goats
}

\begin{abstract}
Early and accurate diagnosis of Brucella melitensis is essential for the treatment and control of brucellosis both in animals and humans. The thrust for the development of a rapid diagnostic technique to overcome the limitations of conventional microbiological and serological tests brought about this investigation on the development and application of dotELISA for antigen and antibody detection in infected goats. Fifteen apparently healthy Boer aged 2-3 years which tested negative for brucellosis using PCR and ELISA, were grouped into A (10 goats infected intraocularly with $107 \mathrm{CFU}$ of B. melitensis) and B (5 goats) as control. Discharges (ocular, nasal, and vaginal) and blood were collected at days 3, 7, 10, 14, weekly until 42 post-infection (pi) for dot-ELISA, PCR, and RBPT. Dot-ELISA detected B. melitensis antigen and antibody in group $\mathrm{A}$ at day 3 and $7 \mathrm{pi}$, respectively with adequate sensitivity and specificity relative to PCR and RBPT. The bacteria shedding detected from discharges at day 3 pi in the nasal and ocular route with dot-ELISA. Group B were consistently negative. Values such as speed, simplicity, field adaptability, high sensitivity, and specificity make dot-ELISA a rapid and adequate technique for diagnosis of brucellosis in B. melitensis infected goats within few hours.
\end{abstract}

Keyword: Brucella melitensis; Dot-ELISA; Diagnosis; Shedding pattern 